\title{
EDITORIAL
}

\section{Vacunación contra influenza en la infancia: una estrategia preventiva de prioridad nacional}

$\mathrm{L}^{2}$ a influenza es una infección altamente contagiosa de origen viral considerada una de las causas más importantes de infecciones de las vías respiratorias. Este padecimiento se asocia a gran morbilidad y mortalidad anualmente. ${ }^{1}$ Sin embargo, esta enfermedad, que se repite año con año, afecta principalmente a poblaciones vulnerables durante los meses de mayor actividad, en el invierno, y en ocasiones se extiende hasta el inicio de la primavera.

México cuenta con un sistema de vigilancia epidemiológica para influenza, que depende del Centro Nacional de Vigilancia Epidemiológica y es operado por el Instituto de Diagnóstico y Referencia Epidemiológicos (InDRE) y por la Dirección General de Epidemiología (DGE). Este sistema nos ha permitido conocer la estacionalidad y el tipo de cepas que circulan año con año en la República Mexicana. Además, dicho sistema ha demostrado que los virus de influenza circulan en México de igual forma como lo hacen en otros países del Hemisferio Norte, produciendo estragos similares. ${ }^{2}$

Todos los segmentos de la población son susceptibles de padecer influenza; pero hasta hace algunos años se consideraba que la infección por estos virus afecta principalmente a la población adulta y ancianos, y a aquellas personas de todos los grupos etarios con enfermedades crónicas subyacentes. ${ }^{1,3-5}$

Sin embargo, datos epidemiológicos recientes han demostrado que, además, la influenza es responsable de ocasionar tasas extraordinariamente elevadas de hospitalizaciones en la edad pediátrica, particularmente en el grupo de 6 a 24 meses. ${ }^{6-8}$ Estas tasas son similares a lo observado en adultos con factores de riesgo de complicaciones y en quienes la vacunación se recomienda anualmente. ${ }^{7,8}$ Está documentado, en algunos países, que la hospitalización por influenza durante la temporada invernal en menores de dos años, y en particular los más pequeños, es igual o mayor que en grupos de alto riesgo y que en adultos sanos de entre 50 y 64 años de edad, en quienes la vacunación anual contra influenza se ha recomendado desde 2000.9-11 La mortalidad atribuible a la enfermedad en niños es mucho menos frecuente que en adultos mayores. La tasa de mortalidad se ha estimado en 3.8 por $100000 .^{7}$

Actualmente, la vacunación anual de personas en grupos de alto riesgo de desarrollar complicaciones, así como sus contactos, representa la principal estrategia para reducir el impacto de la enfermedad. Para este efecto, se utiliza principalmente la vacuna inactivada trivalente por su eficacia y baja reactogenicidad. Según estudios recientes, la vacunación contra influenza puede prevenir $72 \%$ de las hospitalizaciones y $87 \%$ de las muertes provocadas por esta enfermedad. ${ }^{1}$

La vacunación anual contra influenza se recomienda en determinados grupos de individuos con una mayor vulnerabilidad al desarrollo de complicaciones asociadas con esta enfermedad y en aquellos sujetos que pueden transmitirla a personas en grupos de alto riesgo, propensos a desarrollar complicaciones. ${ }^{1}$ Estos grupos son los siguientes:

1. Personal de salud, específicamente a residentes de medicina, internos de hospitales, personal de enfermería y paramédico; personal de salud en servicios de urgencias y en terapias intensivas. De ser posible, vacunación de todo el personal de salud que atiende a pacientes, tanto en centros de salud, hospitales de todos los niveles de atención del sector salud, como en instituciones y consultorios de salud privados.

2. Personal que labora en asilos de ancianos y casas de estancia.

3. Personas que viven en contacto intradomiciliario con otras que tienen alto riesgo de desarrollar com- 
plicaciones (niños que viven con un adulto con asma).

4. Mujeres con embarazo de alto riesgo, específicamente aquellas en el segundo y tercer trimestre del embarazo por tener el mayor riesgo de complicaciones. Este riesgo se documentó durante las pandemias de 1918-1919 y 1957-1958, al encontrarse un exceso de muertes asociadas a influenza en mujeres embarazadas.

5. Personas mayores de 65 años de edad. Este grupo presenta el mayor riesgo de hospitalización y muerte asociado a complicaciones por influenza.

6. Personas de entre 50 a 64 años de edad. En este grupo se concentra la mayor prevalencia de personas con condiciones de alto riesgo (enfermedades crónico-degenerativas).

7. Pacientes de cualquier edad con enfermedades crónicas subyacentes. Estos padecimientos incluyen enfermedades pulmonares (asma o enfermedad pulmonar obstructiva crónica) o enfermedades cardiovasculares (insuficiencia cardiaca). Así como a aquellos con enfermedades metabólicas crónicas (diabetes mellitus, insuficiencia renal) y en pacientes inmunodeprimidos (VIH/SIDA o trasplantados).

8. Viajeros. Se recomienda en aquellos viajeros que no fueron vacunados en el año precedente si planean viajar al trópico o al Hemisferio Sur durante los meses de abril a septiembre.

En octubre de 2003, el Comité Técnico Asesor en Inmunizaciones (ACIP, por sus siglas en inglés) del Centro para la Prevención y Control de las Enfermedades (CDC, por sus siglas en inglés) recomendó la vacunación universal pediátrica al grupo etario de 6 a 23 meses de edad. ${ }^{8}$ De esta forma, la recomendación previa de vacunar a aquellos niños con factores de riesgo para desarrollar complicaciones asociadas con influenza se ha expandido para incluir en forma universal a todos los niños en este grupo poblacional. ${ }^{1,8}$

México, como líder vanguardista en inmunizaciones en el continente americano, ha incluido a la vacunación contra la influenza dentro de sus prioridades. En la segunda reunión extraordinaria del Consejo Nacional de Vacunación (Conava), celebrada el 5 de junio del año 2004, quedó asentado como el acuerdo número nueve, del año en curso que: "las instituciones del Sistema Nacional de Salud iniciarán la vacunación antiinfluenza de manera universal durante la Tercera Semana Nacional de Salud, 2004, los universos se definirán de conformidad con disponibilidad de vacuna".
La única vacuna de influenza licenciada en México para niños menores de cinco años de edad es la trivalente inactivada.

La eficacia de la vacuna en niños sanos ha sido evaluada en diversos ensayos clínicos involucrando a varios miles de aquéllos y realizados durante temporadas de alta actividad de influenza. ${ }^{9-15}$ Estos ensayos clínicos han demostrado que la vacuna inactivada es segura e inmunogénica en niños sanos de 6 meses a 15 años de edad. La eficacia de la vacuna en este grupo de edad varía de entre 31 a 91\% en la prevención de casos confirmados con cultivo de influenza A, y de $45 \%$ contra influenza B. ${ }^{9,12-17}$ En estos estudios también se ha observado una concomitante reducción en los casos de otitis media. ${ }^{15}$ En menores de nueve años de edad, se requiere de dos dosis iniciales para alcanzar el máximo efecto protector. ${ }^{16}$

Entre las acciones de salud que más han beneficiado a los niños de México, destacan las efectuadas por el Programa de Vacunación Universal (PVU). En 1973, un año antes de que entrara en vigor el Programa Ampliado de Inmunizaciones de la Organización Mundial de la Salud (OMS) se estableció en México el Programa Nacional de Inmunizaciones, con la aplicación de los mismos seis biológicos incluidos en las vacunas BCG, OPV, DPT y antisarampión. No se habían efectuado cambios en el esquema básico de vacunación de México hasta 25 años después, en 1998, con la inclusión de las vacunas triple viral (SRP) y nuevamente, en 1999, con la introducción de la vacuna pentavalente (DPT-Hib-HB), cambios que pusieron a México a la vanguardia, en relación con la vacunación universal, y permitieron que por primera vez en su historia las coberturas en niños de un año de edad rebasaran 95\% en 2003.

El extender la vacunación anual contra influenza en la edad pediátrica, dirigiéndose principalmente al grupo de edad de mayor riesgo, representa un gran reto para la salud pública nacional. Aunque la vacuna ofrece la posibilidad de prevenir una importante proporción de infecciones virales durante el invierno, otras como las ocasionadas por el virus sincicial respiratorio y otros patógenos respiratorios continuarán ocurriendo. La educación de los padres y del personal de salud es, sin duda alguna, un componente integral del programa pediátrico de vacunación contra influenza. Esta estrategia muy probablemente demostrará ser de costo-beneficio $^{17,18}$ por la disminución en la morbilidad y mortalidad en la edad pediátrica y por su efecto de inmunidad de rebaño en la población adulta, como ha sido demostrado en Japón..$^{19,20}$ 
La inclusion de la vacuna contra influenza en población pediátrica de 6 a 23 meses y en adultos mayores es una decisión acertada. Sin embargo, esta política de salud pública requiere de los recursos suficientes para poder asegurar: la compra anual del biológico, las coberturas en la población blanco, la vigilancia epidemiológica correspondiente, incluyendo la de eventos temporalmente asociados a vacunación (ETAV), y que la sustentabilidad del Programa de Vacunación Universal, como bien público, esté garantizado con una ley sobre vacunas.

José Ignacio Santos Preciado, MD, MSc, * Carlos Franco Paredes, MC, MPH. $\ddagger$

\section{Referencias}

1. Centers for D isease Control and Prevention. Prevention and Control of Influenza. Recommendations of the Advisory Committee on Immunization Practices (ACIP). MMW R Morb Mortal W kly Rep 2004; 53(RR06):1-40.

2. Secretaría de Salud. Dirección General de Epidemiología:Anuarios de Morbilidad 1999-2001. Disponible en: http://www.dgepi.com.mx. 3. G ensheimer KF, Meltzer MI, Postema AS, Strikas R. Influenza pandemic preparedness. Emerg Infect D is 2003;9(12):1645-1648.

4. Centers for Disease Control and Prevention. U pdate: Influenza activity-United States, 2003-2004 Season. MMW R Morb Mortal W kly Rep 2003;52(49):1197-1202.

5. D emicheli V, Jefferson T, Rivetti D, D eeks J. Prevention and early treatment of influenza in healthy adults. Vaccine 2000;18:957-1030. 6. O 'Brien KI,W alters MI, Sellman J, Q uinlisck P, Regnery H, Schwartz B et al. Severe pneumonia in previously healthy children:The role of preceding influenza infection. C lin Infect D is 2000;30:784-789. 7. Mulloly JP, Barker W H. Impact of type A influenza on children:A retrospective study.Am J Public Health 1982;72:1008-1016. 8. Centers for Disease Control and Prevention. Prevention and control of influenza. Recommendations of the Advisory Committee on
Immunizations Practices (ACIP) MMW R Morb Mortal W kly Rep 2003;52(RR-8):1-34.

9. Hurwitz ES, Haber M, C hang A, Shope T, Teo ST, Giesck JS et al. Studies of the 1996-1997 inactivated influenza vaccine among children attending day care: Immunologic response, protection against infection, and clinical effectiveness. J Infect D is 2000;182:1218-1221.

10. Bernstein DI, Zahradnik JM, D eA gnelis CJ, C herry JD. C linical reactions and serologic responses after vaccination with whole-virus or split-virus influenza vaccines in children aged 6 to 36 months. Pediatrics 1982;69:404-408.

11. W hite T, Lavoie S, N ettleman MD. Potential cost savings attributable to influenza vaccination of school-aged children. Pediatrics 1999;103(6):E73.

12. Clements $D A$, Langdon $L, B$ land $C, W$ alter E. Influenza $A$ vaccine decreases the incidence of otitis media in 6- to 30-month-old children in day care.Arch Pediatr Adolesc Med 1995;149:1113-1117.

13. Kramarz P, D eStefano F, Gargiullo PM, D avis RL, Chen RT, Mullooly JP et al. Influenza vaccination in children with asthma in health maintenance organizations. Vaccine Safety D atalink Team.Vaccine 2000;18:2288-2294

14. N euzil KM, D upont W D, W right PF, Edwards KM. Efficacy of inactivated and cold-adapted vaccines against influenza A infection, 1985 to 1990:The pediatric experience. Pediatr Infect D is I 2001;20:733-740. 15. H oberman A, G reenberg D P, Paradise JL, Rockette HE, Lare JR, Kearney DH et al. Effectiveness of inactivated influenza vaccine in preventing acute otitis media in young children:A randomized controlled trial. JAMA 2003;290:1608-1616.

16. Ruben FR. Inactivated influenza virus vaccines in children. C lin Infect $D$ is 2004;38:678-688.

17. N euzil KM. Influenza vaccine for children. C lin Infect $D$ is 2004:38:689-690.

18. Luce BR, Zangwill KM, Palmer CS, Mendelman PM, Yan L,W olff MC et al. Cost-effectiveness analysis of an intranasal influenza vaccine for the prevention of influenza in healthy children. Pediatrics 2001;108(2):E24.

19. Reichert TA, Sugaya N , Fedson DS, G lezen W P, Simonsen L, Tashiro M et al.The Japanese experience with vaccinating schoolchildren against influenza. N Engl J Med 2001;344:889-896.

20. Reichert TA. The Japanese program of vaccination of schoolchildren against influenza: Implications for control of the disease. Semin Pediatr Infect D is 2002;13(2):104-111.

\footnotetext{
* Director General del Hospital Infantil de México.

‡ Hospital Infantil de México Federico Gómez.
} 